\title{
Reaching for the unreachable: integration of locomotion and whole-body movements for extended visually guided reaching
}

\author{
Martim Brandão, Lorenzo Jamone, Przemyslaw Kryczka, Nobotsuna Endo, Kenji Hashimoto, Atsuo Takanishi
}

\begin{abstract}
We present a novel control architecture for the integration of visually guided walking and whole-body reaching in a humanoid robot. We propose to use robot gaze as a common reference frame for both locomotion and reaching, as suggested by behavioral neuroscience studies in humans. A gaze controller allows the robot to track and fixate a target object, and motor information related to gaze control is then used to i) estimate the reachability of the target, ii) steer locomotion, iii) control whole-body reaching. The reachability is a measure of how well the object can be reached for, depending on the position and posture of the robot with respect to the target, and it is obtained from the gaze motor information using a mapping that has been learned autonomously by the robot through motor experience: we call this mapping Reachable Space Map. In our approach, both locomotion and whole-body movements are seen as ways to maximize the reachability of a visually detected object, thus i) expanding the robot workspace to the entire visible space and ii) exploiting the robot redundancy to optimize reaching. We implement our method on a full 48-DOF humanoid robot and provide experimental results in the real world.
\end{abstract}

\section{INTRODUCTION}

One of the ultimate goals of humanoid robots is to be able to walk around and perform different kind of actions in the human environment. The humanoid structure combines the possibility to locomote in a human designed environment to the one of interacting with objects through reaching and, possibly, fine manipulation. However, despite the recent advancements in robot design and in the control of locomotion, only a few implementations exist in which walking behavior is integrated with complex reaching abilities, and in which these actions are autonomous, goal directed and driven by robot vision. This is clearly a fundamental prerequisite to having humanoid robots seamlessly included in our society.

In recent works [1], [2] we explored the concept of reachability, and we proposed what we call the Reachable

* Study conducted as part of the Research Institute for Science and Engineering, Waseda University, and humanoid project at Humanoid Robotics Institute, Waseda University; supported in part by MEXT/JSPS KAKENHI (Grant Number: 24360099 and 25220005), and Strategic Young Researcher Overseas Visits Program for Accelerating Brain Circulation, JSPS, Japan.

M. Brandão is with the Graduate School of Advanved Science and Engineering, Waseda University, 41-304, 17 Kikui-cho, Shinjuku-ku, Tokyo 1620044, JAPAN. contactetakanishi.mech. waseda.ac.jp

L. Jamone is with Instituto Superior Técnico (ISR/IST), Portugal.

P. Kryczka is with the Graduate School of Advanved Science and Engineering, Waseda University.

N. Endo is with Dept. of Adaptive Machine Systems, Osaka University.

$\mathrm{K}$. Hashimoto is with the Faculty of Science and Engineering, Waseda University.

A. Takanishi is with the Department of Modern Mechanical Engineering and director of the Humanoid Robotics Institute, Waseda University.
Space Map (RSM). The reachability is a measure of how well a $3 \mathrm{D}$ location in space can be reached for, depending on the position and posture of the robot with respect to that location; it is not just binary information (reachable/non-reachable), but a measure of the expected quality of the reaching action, based on the robot's previous motor experience. We proposed to encode this information directly in the robot motor space, and in particular using the motor variables that are involved in gaze control (i.e. joints of the neck and eyes). The RSM is a mapping that is learned autonomously by the robot from motor experience, during the execution of goal-directed reaching movements: this mapping relates the current gaze configuration of the robot to the reachability of the fixated 3D point in space. Therefore, the RSM can be used to estimate the reachability of a fixated object, and to plan whole-body movements that increase its reachability, allowing to reach for the object in a better way; we showed this in previous simulation results [2].

Starting from this concept, here we propose a novel control architecture in which both locomotion and wholebody movements are seen as ways to increase the reachability of a fixated object, thus realizing a form of autonomous, goal directed and visually guided behavior that incorporates locomotion and whole-body reaching. The behavior is reactive, and fundamentally based on the control of gaze, which serves as a main reference. The robot head is controlled to gaze at a visually detected target object, and then everything follows: the robot walks following the gaze direction, the gaze-encoded reachability is maximized through walking and whole-body movements, and finally the gaze configuration is used as a reference for the control of arm reaching.

The rest of the paper is organized as follows. After briefly reviewing the related work in Section II, in Section III we present the robotic platform on which we implemented our system. Then the whole control architecture is described in Section IV. Finally, we show the experimental results in Section V, while in Section VI we report our conclusions and we sketch the future work.

\section{RELATED WORK}

Behavioral studies on humans enforce the hypothesis of the presence of a gaze-centered reference frame for the control of reaching [3] and whole-body reaching [4]. Moreover, recent studies have assessed the nature of the visual strategies governing the steering of locomotion [5] and shown that head angle anticipates walking direction even in the darkness [6]. 
These findings suggest that humans rely on a gaze-centered control of both locomotion and reaching: on the other hand, most humanoid robotics approaches attempt at solving these control problems through Cartesian global planning. One example is [7], where whole-body reaching postures are computed through inverse kinematics and footsteps planned to avoid obstacles and achieve such a final configuration. Other works focusing on global planning of whole-body motion include for example [8] focusing on collision avoidance or [9] which formulates step planning as part of a virtual kinematic chain whose inverse kinematics with constraints are solved numerically. In [10], heuristics inspired from human motion are proposed for planning of the walk-thenreach task. Which foot to move, step size and angle, head direction, among others, are decided based on probability distributions of observations taken from human motion data.

However, global planning is computationally expensive and can be difficult to implement in real-time. Moreover, planning in Cartesian space requires the computation of the transformations from motor/sensory space to Cartesian space: this computation is typically affected by some level of noise and inaccuracy, that may deteriorate the system's performance in the real world. Opposed to global planning, and adopted in the present paper, reactive control based on visual servoing is an approach which can be easily executed in real-time. A notable example is described in [11], where the tasks of visual servoing and grasping are done during locomotion. The stack of tasks (SoT) framework is used, which enables several tasks to be executed given a certain task priority, lower level tasks being kept from interfering with higher level ones through the use of consecutive nullspace projections. Using this framework, the work described in [11] deals with arm reaching during locomotion; however, whole-body reaching is not considered, thus limiting the robot's range of action. Similarly to [11], visual servoing and reaching is also tackled in [12], where reachability is considered by selection of the best reaching strategy out of a fixed set (e.g. reaching with the left or right hand).

The main contribution of the present paper with respect to the works in [11], [12] is the use of the RSM to control the position and posture of the robot before reaching is executed, thus optimizing the reaching task based on the estimated reachability. Exploiting the robot redundancy by controlling the waist in an optimal way remarkably expands the robot's potential, allowing also robots with a limited range of motion of the arms to perform reaching within a big workspace. Moreover, as we do not deal at all with Cartesian coordinates, but only with motor variables (in particular, the ones involved in the gaze control are the main reference for both control and optimization of the whole system), we don't need to compute the transformations from motor space and visual space to Cartesian space. Then, in this paper we perform reaching with the right arm, but our approach is general and can be easily extended to bimanual reaching, as we showed in previous simulation work [2].

\section{THE HUMANOID PLATFORM}

The robotic platform we use in this work is the 48 degrees of freedom (DOF) humanoid robot KOBIAN [13]. The robot size is similar to that of an average Japanese woman (see left image in Figure 1) and its weight is $62 \mathrm{~kg}$. The DOF of the robot are distributed as follows: 12 in the legs, 3 in the waist, 14 in the arms, 8 in the hands, 4 in the neck and 7 in the head (see right image in Figure 1). Joints are controlled by a PC mounted on the robot's back, which in turn is a node of a local network of several PCs. This network is exploited to perform distributed computation, supported by the use of YARP [14]. Two color cameras provide 640x480 pixel images at a frame rate of $30 \mathrm{~Hz}$.
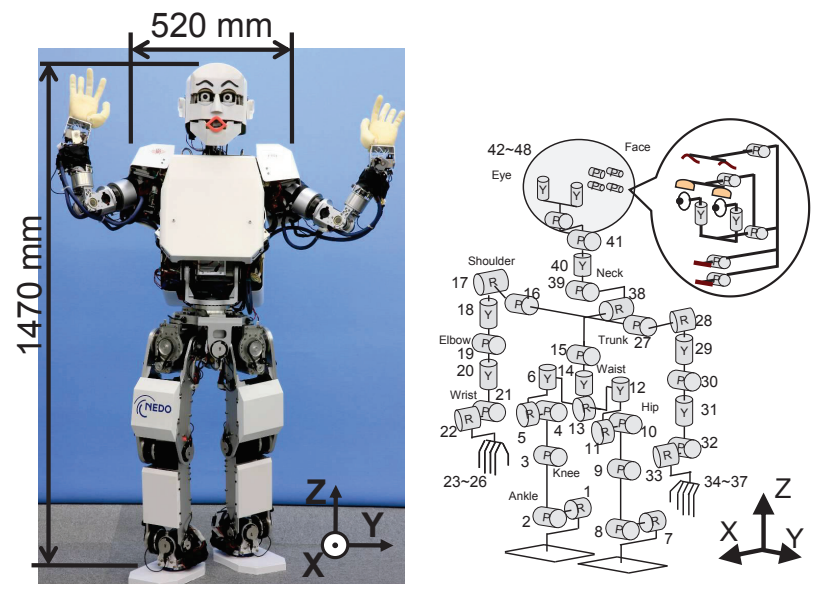

Fig. 1. KOBIAN humanoid robot. Left image: KOBIAN expressing surprise. Right image: description of the robot 48 DOFs.

As we perform visually-guided locomotion and wholebody reaching, we aim at controlling the KOBIAN head $\left(\mathbf{q}_{\text {gaze }}=\left[\begin{array}{lll}\theta_{y} & \theta_{p} & \theta_{v}\end{array}\right] \in \mathbb{R}^{3}\right.$ : yaw and pitch of the neck, vergence of the eyes), arm $\left(\mathbf{q}_{\text {arm }} \in \mathbb{R}^{4}\right.$ : shoulder and elbow of the right arm), waist ( $\mathbf{q}_{\text {waist }} \in \mathbb{R}^{3}$ : yaw, pitch and roll) and legs $\left(\mathbf{q}_{\text {rleg }} \in \mathbb{R}^{6}\right.$ and $\left.\mathbf{q}_{\text {lleg }} \in \mathbb{R}^{6}\right)$. The robot endeffector is represented by a visual marker (a green ball) that is attached to the wrist (as a marker for the hand); in the experiments, the target of the reaching movements is a red ball. The position of the red and green balls' center in the camera images is computed with a precision of about \pm 2 pixels, using a combination of top-down [15] and bottom-up [16] tracking techniques for extra motion robustness that we described in [16].

\section{Control ARCHitecture}

We illustrate here the different components of the control. A gaze controller (described in IV-A) guarantees tracking and fixation of the target, and together with the reachability estimation (obtained by querying the RSM as described in IV-B) provides the necessary information to drive the other controllers: a waist controller (described in IV-C) that maximizes the reachability of the target by finding an optimal configuration of the body, a locomotion controller (described in IV-D) that maximizes the reachability by moving towards 
the target when it is too far (or back if the target is too close), steering with a "follow gaze direction" control approach, and a controller for arm reaching that uses the gaze configuration as a reference, as described in IV-E.

The ensemble of these controllers generates a goaldirected autonomous behavior in which the robot follows the target in a reactive manner (i.e. no computationally expensive planning is involved), fully exploiting its high level of redundancy (i.e. walking, rotating and bending the torso) to eventually reach for the target object in an optimal way with respect to the estimated reachability.

\section{A. Gaze control}

The gaze controller allows to track and to eventually fixate a target (i.e. a 3D point in space) by moving the head and eyes. If the target is visible (i.e. inside the image plane) joints velocities are generated as follows:

$$
\dot{\mathbf{q}}_{g a z e}(t)=-G \mathbf{x}(t)
$$

where $G \in \mathbb{R}^{3 \times 3}$ is a positive definite gain matrix and the position of the target $\mathbf{x} \in \mathbb{R}^{3}$ is defined as follows:

$$
\mathbf{x}=\left[\begin{array}{c}
u_{L}-u_{R} \\
\left(u_{L}+u_{R}\right) / 2 \\
\left(v_{L}+v_{R}\right) / 2
\end{array}\right]=\left[\begin{array}{cccc}
1 & -1 & 0 & 0 \\
1 / 2 & 1 / 2 & 0 & 0 \\
0 & 0 & 1 / 2 & 1 / 2
\end{array}\right]\left[\begin{array}{c}
u_{L} \\
u_{R} \\
v_{L} \\
v_{R}
\end{array}\right]
$$

being $\left(u_{R}, v_{R}\right)$ and $\left(u_{L}, v_{L}\right)$ the coordinates of the target on the right and left image plane respectively.

If the target is not visible a stereotyped motion strategy (i.e. random left-right and up-down movements of the neck) is used to detect it; then the controller (1) is activated. More details about this gaze controller can be found in previous publications [17], [2]. After fixation is achieved we encode the target position in space using the gaze configuration $\mathbf{q}_{\text {gaze }}$. Since we actuate only 3 DOF of the head+eyes system the mapping from gaze configuration to target position is unique, but if more DOF are used the redundancy should be solved by the gaze controller, as for instance in [18]. This motor information will be then used as a reference for all the other controllers described hereinafter.

\section{B. Reachable space map (RSM)}

The RSM is a mapping that defines the reachability of a fixated object (i.e. the reachability of the fixated location in space) as a function of its position with respect to the robot, as follows:

$$
R=f_{R S}\left(\mathbf{q}_{\text {gaze }}\right),
$$

where the object position is encoded using the gaze configuration $\mathbf{q}_{\text {gaze }}$. The robot learns this mapping incrementally using LWPR [19], an online algorithm for non-linear regression that provides a compact representation and can be queried fast in real-time. After each reaching action, data is collected in the form $\left(\mathbf{q}_{\text {gaze }} ; R\right)$, where $\mathbf{q}_{\text {gaze }}$ is the gaze configuration defining the target object position and $R \in[0,1]$ is a continuous value that represents an evaluation of the action: if the target is not reached $R$ is in inverse proportion to the final visual error of the arm reaching (ranging from 0 to 0.5 ),

while if it is reached $R$ is proportional to the "optimality" of the final arm configuration (ranging from 0.5 to 1.0). In our system we defined this "optimality" as the distance of the arm from the joint limits.

Further details about how this mapping is defined and learned can be found in [2], applied to a different simulated robot. To realize the experiments described in this paper, KOBIAN has learned its RSM while performing 500 arm reaching attempts toward target objects randomly placed around itself. The normalized mean squared error (NMSE) of the estimation has been computed with respect to a given test set of $250\left(\mathbf{q}_{\text {gaze }}, R\right)$ samples, not used for training: the NMSE is 0.35 after the first 25 reaching attempts, then it drops to 0.17 (after 50 attempts), to 0.10 (after 200 attempts), and eventually to 0.08 (after 500 attempts). Figure 2 shows a visual representation of the RSM of KOBIAN after 500 reaching attempts. The output of the RSM is smooth and continuous, as seen from the smooth color gradient in the plots: this is an important characteristic since we want to use the RSM for conrol (as described in Section IV-C).
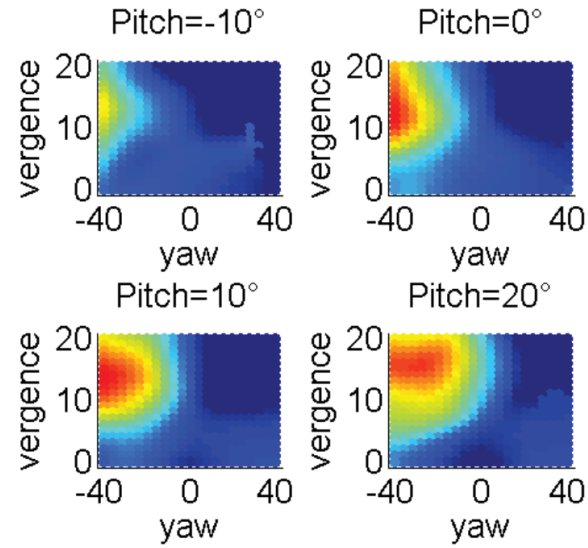

Fig. 2. KOBIAN's RSM after 500 reaching actions. Four 2D projections on the yaw/vergence place, with different values of pitch, namely: pitch $=$ $\left[-10^{\circ} ; 0^{\circ} ; 10^{\circ} ; 20^{\circ}\right]$. The color bar indicates the relation between color and reachability value: from 0 , blue color, to 1 , red color.

\section{Waist control}

The motion of the waist is used to both i) extend the robot tracking capabilities, that can be limited by the head joint limits if only the controller (1) is used, and ii) place the robot in an optimal configuration with respect to the object (i.e. maximizing the reachability). These two tasks are achieved using two separate controllers given by (3) and (6).

If during gaze control $\mathbf{q}_{\text {gaze }}$ reaches the limits, the waist is used to extend the robot range of action, as follows:

$$
\dot{\mathbf{q}}_{\text {waist }}=-G_{w} \mathbf{x}
$$

where $G_{w} \in \mathbb{R}^{3 x 3}$ is a positive definite gain matrix.

If instead $\mathbf{q}_{\text {gaze }}$ is within the limits, the tracking (i.e. fixating) task can be accomplished actuating only the $\mathbf{q}_{\text {gaze }}$ joints using (1), and therefore the waist joints can be used to position the robot in an optimal configuration with respect 
to the object. To realize this task, first we compute the head and eyes velocity that would realize a desired change in the reachability. To do this, we exploit the pseudo-inverse of the Jacobian of the RSM, which is obtained by differentiating (2), as follows:

$$
\begin{gathered}
J_{R S}\left(\mathbf{q}_{g a z e}\right): \dot{R}=J_{R S}\left(\mathbf{q}_{\text {gaze }}\right) \dot{\mathbf{q}}_{\text {gaze }} \\
\dot{\mathbf{q}}_{\text {gaze }}^{d}=J_{R S}^{\dagger}\left(\mathbf{q}_{\text {gaze }}\right) d R^{d}
\end{gathered}
$$

where $d R^{d}$ is the desired change in the reachability, which is typically chosen as the difference between the maximum reachability $\left(R_{\max }=1.0\right)$ and the current one $R$, leading to $d R^{d}=R_{\max }-R$. Then, from the desired head and eyes motion $\dot{\mathbf{q}}_{\text {gaze }}^{d}$ we derive a motion of the body $\dot{\mathbf{q}}_{b}$ that, if executed, would cause the head and eyes to follow the desired motion $\dot{\mathbf{q}}_{\text {gaze }}^{d}$ because of the gaze controller (1). This transformation from head and eyes motion to body motion is obtained exploiting an approximated kinematic model,

$$
\dot{\mathbf{q}}_{\text {waist }}=K_{b h} \dot{\mathbf{q}}_{g a z e}^{d}
$$

where $K_{b h} \in \mathbb{R}^{3 x 3}$ is the constant matrix defining the model. The idea behind this controller is that the robot redundancy can be exploited replacing the motion of the head and eyes joints with the motion of the waist.

\section{Locomotion control}

The reachability information can also be used to trigger locomotion of the robot towards the target in a similar way to (6): where vergence is directly controlled by locomotion in the direction that increases reachability. This way, a reference walking progression speed $W_{\text {ref }}$ (positive or negative for forward or backward walking) can be computed from the RSM,

$$
W_{\text {ref }}=K_{\text {loco }} \dot{\mathbf{q}}_{\text {gaze }}^{d}(2),
$$

where $K_{\text {loco }} \in \mathbb{R}$ is a constant relating desired vergence to cartesian speed and $\dot{\mathbf{q}}_{\text {gaze }}^{d}(2)$ the vergence component of the desired gaze velocity obtained from (5). On the other hand, the direction that increases reachability is naturally the gaze direction when the target is fixated or the robot would deviate from that target. Practically locomotion steering can hence be implemented by a gaze-centered locomotion control inspired by the "locomotion follows gaze" finding [6] as to center gaze with respect to the body.

We define the orientation of the robot waist in the world as $\alpha(t)$ and its average rate of change as $\alpha^{\prime}(t)=\alpha(t+$ $\Delta s)-\alpha(t)$ where $\Delta s$ is the duration of a step. At time $t$, footsteps $t$ and $t-\Delta s$ are on the floor, and the swing foot's motion is defined by $\theta_{f t}^{\prime}(t)=\theta_{f t}(t+\Delta s)-\theta_{f t}(t-\Delta s)$. Swing foot displacement is fully defined by $W_{\text {ref }}$ and $\theta_{f t}^{\prime}(t)$ by assuming that at the middle of the double support phase the waist lies in the middle of the two feet.

A gaze-centered control basically steers walking direction to bring the yaw component of $\mathbf{q}_{\text {gaze }}$ to zero,

$$
\alpha^{\prime}(t)=-G_{\text {loco }} \cdot \mathbf{q}_{\text {gaze }}(0),
$$

where $G_{\text {loco }} \in \mathbb{R}$ is a control gain. Satisfying $\alpha^{\prime}(t)$ could imply feet collision or reaching joint limits at the current desired progression speed $W_{\text {ref }}$ and so measures must be taken as to scale down $W_{\text {ref }}$ and $\alpha^{\prime}(t)$ to avoid such situation. Given $W_{\text {ref }}$, the goal is then to compute $\theta_{\text {ftsafe }}^{\prime}(t)$ and $\alpha_{s a f e}^{\prime}(t)$ leading to the maximum real progression speed $W_{\text {safe }} \leq W_{\text {ref }}$ that still avoids joint limits and self-collision. The following optimization problem needs to be solved:

$$
\max _{\substack{\text { feetcollision }\left(\theta_{f t}^{\prime}(t)\right)=0 \\ A x \leq b}} W,
$$

where $W$ is the executed progression speed and feetcollision $\left(\theta_{f t}^{\prime}(t)\right)$ indicates whether a swing foot motion that satisfies $\theta_{f t}^{\prime}(t)$ without collisions exists. The optimization variable is defined as $\mathbf{z}=\left[\begin{array}{ll}\alpha^{\prime} & \theta_{f t}^{\prime}\end{array}\right]$, while $A$ and $b$ are set as to satisfy leg joint limits $\left(\mathbf{q}_{\text {lleg }}, \mathbf{q}_{\text {rleg }}, \alpha^{\prime}\right)$ and progression limits ( $W \leq W_{\text {ref }}$ ). One possible solution to this optimization is to select a foot transition from a set of safe foot trajectories which is available a priori. This is a usual approach in footstep planning works that plan feet trajectories on a $A^{*}$ algorithm given a small set of feet transitions [20]. For simplicity, in this work we set a heuristic where turning speed is proportional to progression speed and the feet are parallel to the waist, as follows:

$$
\begin{aligned}
& \alpha_{\text {safe }}^{\prime}(t)=\alpha^{\prime}(t) \cdot W_{\text {safe }} / W_{\text {ref }} \\
& \theta_{\text {ftsafe }}(t+1)=\alpha_{\text {safe }}(t+1) .
\end{aligned}
$$

Compared to an $\mathrm{A}^{*}$ approach, such heuristics allow for high precision of movement since transitions are not discretized, at the extra cost of feet collision computation. Feet collision is in our implementation computed by intersection of the two feet as 2D polygons. The whole-body, dynamically stable, locomotion pattern is then generated from (7) and (10) through use of the FFT-based pattern generator described in [21], [22]. The pattern generator computes a compensation of the torso trajectory, in a multibody system dynamics approach, leading to the desired ZMP trajectory. ZMP reference trajectories were set as to lie on the stance foot's center during the whole swing phase and to smoothly shift (through spline interpolation) to the other feet during double support phase. A feedback controller is active during the whole procedure, which compensates for disturbances of the ZMP with respect to the reference.

\section{E. Arm reaching control}

The approach we use for controlling arm reaching was initially proposed in [17], and slightly modified in [2]. The version we use here is the same as [2], but applied to the KOBIAN robot. The reader can refer to those publications to get detailed descriptions of the controller. Two kinematic models (that are learned online during the movements) are employed in the control. A motor-motor mapping $\mathbf{q}_{\text {gaze }}=$ $f_{F K}\left(\mathbf{q}_{\text {arm }}\right)$ relates the gaze configuration to the arm position which brings the end-effector (i.e. robot hand) to the fixation point: inverting this function allows to retrive the desired arm position to reach for the target in an open-loop fashion. Then an additional closed-loop controller based on visual servoing is used to eliminate small position errors when the hand is close to the target. 


\section{EXPERIMENTAL RESULTS}

We validated the proposed methodology by performing a series of locomotion and whole-body reaching trials using the KOBIAN robot. Trajectories of all joints (i.e. joint encoder values) and reachability $R$ of the target were recorded with a sampling rate of $50 \mathrm{~Hz}$. In each trial a target object is placed far away from the robot, at a distance between one and three meters. The gaze controller is always active for the whole duration of the experiment. After the initial fixation of the target, the low level of reachability obtained from the RSM, combined with the observation of a small value of vergence (i.e. fixated target far from the robot), triggers the activation of the walking controller. Then the robot walks keeping fixation of the target, until the vergence reaches a threshold value. Then the whole-body reaching action starts: the robot moves the waist to find an optimal posture with respect to the target object, and then the arm reaching movement is executed. We measure the outcome of the reaching action in terms of optimality of the final arm configuration (i.e. distance from joint limits), to prove that through the use of the RSM the robot is not only able to successfully reach for the targets, but it does so in an optimal way.

TABLE I

REACHABILITY $R$ IN 10 TRIALS

\begin{tabular}{cccc}
\hline & Beginning & After locomotion & After waist control \\
\hline \hline Mean & 0.37 & 0.48 & 0.67 \\
Std-dev & 0.02 & 0.07 & 0.07 \\
\hline
\end{tabular}

One of the trials is shown in Figures 3 and 4 as an example. By comparing the reachability and joint trajectories, the effects of each controller on target reachability become clear: while locomotion mainly acts on the vergence component of gaze to bring the target closer to the robot, it can only bring $R$ so close to the reachable-unreachable threshold ( $R=0.5$ indicates the target can be reached with zero visual error). The waist controller then compensates for the low range of movement of the arms and is the main responsible for optimizing the reaching task since most of the increase in $R$ occurs during waist activation. This intuitive result comes from the kinematic constraints of the robot (e.g. height during natural locomotion, arms' low range of movement), which for most target positions makes the use of waist motion mandatory for a successful reach. By comparing the gaze trajectories with the RSM of KOBIAN in Figure 2, it is also clear that the trajectories are consistent with the map and follow a path to its maximum. A sequence of images of the robot during this trial is shown in Figure 4.

For all 10 trials the robot managed to reach for the target. On average, as seen in Table I, with locomotion the target was brought from an initially unreachable state to a position which is reachable with low visual error ( $R$ close to 0.5 ). The final $R=0.67$, if interpreted in terms of the distance of the joints from $\left(q_{\max }+q_{\min }\right) / 2$, corresponds to an average normalized distance of $M=0.66$ - meaning the arm joints are still far from reaching their limits $(M=1)$.
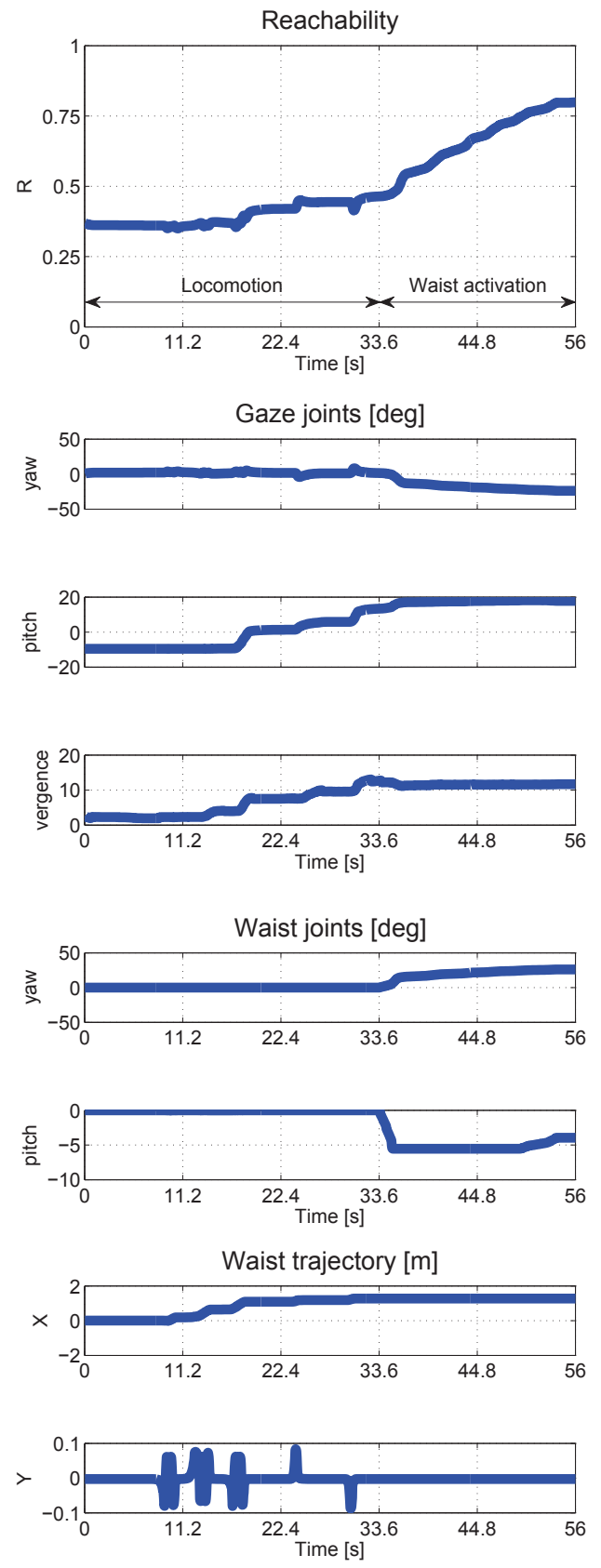

Fig. 3. Trajectories of $R$, gaze and waist angles and waist position in space during one whole-body reaching trial. Target is reached at $t=56$.

\section{CONCLUSIONS AND FUTURE WORK}

We presented, implemented and validated a novel control architecture for gaze-centered whole-body reaching and locomotion. The proposed architecture enables a robot with restricted arm workspace (as it can be noticed in Figure 2) to reach for targets initially unreachable: not only with a successful reach in all experimental trials but also in an optimal way in terms of distance of the arm joints from their limits. This result is achieved in a real scenario using a reactive, local control that exploits the RSM representation. Because of its definition in the motor space and its 

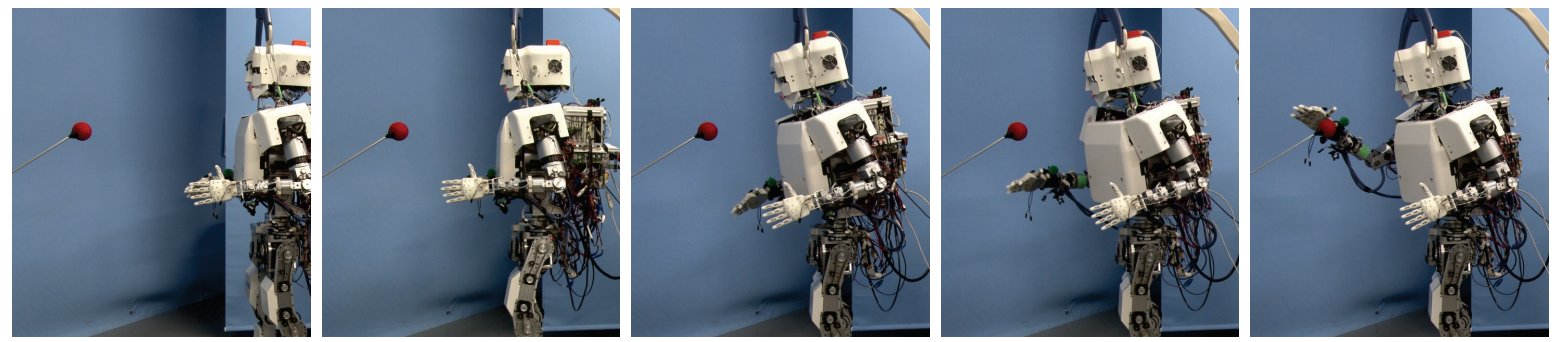

Fig. 4. Sequence of images taken during one of the integrated locomotion and whole-body reaching experiments $(t=14,24,34,54,56)$.

compact representation, the RSM proves to be well suited for online control of a complex task such as whole-body reaching. Future work will focus on extensions to reaching with obstacle avoidance: this could be done by fixating the obstacles and representing them as repulsive fields in the RSM map (similarly to [23]). Then, other interesting research directions include the gaze-encoded prediction of target motion, that would allow dynamic reaching-whilewalking with moving targets, and the gaze-encoded planning of locomotion viapoints, that would allow to avoid obstacles during both walking and reaching. To cope with these more complex scenarios, the reactive approach proposed here might be combined with planning: one possibility could be to exploit both approaches together with different control rates, the latter being realized at a slower rate than the former. Indeed, although not yet explored in this work, the RSM can be used not only for local control (e.g. gradient descent like in the present paper) but also global planning. Because of its compact map representation, planning in RSM space is promisingly more computationally efficient than traditional geometric and kinematic planning. As opposed to local techniques, global planning in RSM space could possibly improve even further the optimality of the reaching movements as it allows to avoid local maxima. In the future we will explore this planning paradigm, as well as more extensively validate gaze-centered locomotion.

\section{REFERENCES}

[1] L. Jamone, L. Natale, K. Hashimoto, G. Sandini, and A. Takanishi, "Learning the reachable space of a humanoid robot: a bio-inspired approach," in International Conference on Biomedical Robotics and Biomechatronics. IEEE, 2012.

[2] L. Jamone, L. Natale, G. Sandini, and A. Takanishi, "Interactive online learning of the kinematic workspace of a humanoid robot," in International Conference on Intelligent Robots and Systems, 2012.

[3] K. Fiehler, I. Schtz, and D. Y. P. Henriques, "Gaze-centered spatial updating of reach targets across different memory delays," Vision Research, vol. 51, pp. 890-897, 2011.

[4] M. Flanders, L. Daghestani, and A. Berthoz, "Reaching beyond reach," Experimental Brain Research, vol. 126, no. 1, pp. 19-30, 1999.

[5] R. M. Wilkie, J. P. Wann, and R. S. Allison, "Active gaze, visual lookahead, and locomotor control," Journal of Experimental Psychology: Human Perception and Performance, vol. 34, no. 5, pp. 1150-1164, 2008.

[6] P. Prévost, I. Yuri, G. Renato, and B. Alain, "Spatial invariance in anticipatory orienting behaviour during human navigation," Neuroscience letters, vol. 339, no. 3, pp. 243-247, 2003.

[7] E. Yoshida, C. Esteves, O. Kanoun, M. Poirier, A. Mallet, J.-P. Laumond, and K. Yokoi, "Planning whole-body humanoid locomotion, reaching, and manipulation," in Motion Planning for Humanoid Robots. Springer, 2010, pp. 99-128.
[8] S. Dalibard, A. Nakhaei, F. Lamiraux, and J.-P. Laumond, "Wholebody task planning for a humanoid robot: a way to integrate collision avoidance," in Humanoid Robots, 2009. Humanoids 2009. 9th IEEERAS International Conference on. IEEE, 2009, pp. 355-360.

[9] O. Kanoun, J.-P. Laumond, and E. Yoshida, "Planning foot placements for a humanoid robot: A problem of inverse kinematics," International Journal of Robotics Research, vol. 30, no. 4, pp. 476-485, 2011.

[10] M. Sreenivasa, P. Souères, and J. Laumond, "Walking to grasp: Modeling of human movements as invariants and an application to humanoid robotics," Systems, Man and Cybernetics, Part A: Systems and Humans, IEEE Transactions on, vol. 42, no. 4, pp. 880-893, 2012.

[11] N. Mansard, O. Stasse, F. Chaumette, and K. Yokoi, "Visuallyguided grasping while walking on a humanoid robot," in International Conference on Robotics and Automation. IEEE, 2007, pp. 3041-3047.

[12] M. Gienger, B. Bolder, M. Dunn, H. Sugiura, H. Janssen, and C. Goerick, "Predictive behavior generationa sensor-based walking and reaching architecture for humanoid robots," in Autonome Mobile Systeme 2007. Springer, 2007, pp. 275-281.

[13] N. Endo and A. Takanishi, "Development of whole-body emotional expression humanoid robot for adl-assistive rt services," Journal of Robotics and Mechatronics, vol. 23, no. 6, pp. 969-977, 2011.

[14] G. Metta, P. Fitzpatrick, and L. Natale, "Yarp: yet another robot platform," International Journal on Advanced Robotics Systems, vol. 3, no. 1, pp. 43-48, 2006, special Issue on Software Development and Integration in Robotics.

[15] M. Taiana, J. Santos, J. Gaspar, J. Nascimento, A. Bernardino, and P. Lima, "Tracking objects with generic calibrated sensors: an algorithm based on color and 3d shape features," Robotics and Autonomous Systems, vol. 58, no. 6, pp. 784-795, 2010.

[16] M. Brandão, A. Bernardino, and J. Santos-Victor, "Image driven generation of pose hypotheses for 3d model-based tracking," in IAPR Conference on Machine Vision Applications, 2011, pp. 59-62.

[17] L. Jamone, L. Natale, G. Metta, F. Nori, and G. Sandini, "Autonomous on-line learning of reaching behavior in a humanoid robot," International Journal of Humanoid Robotics, vol. 9, no. 3, p. 1250017, 2012.

[18] L. Natale, F. Nori, G. Metta, and G. Sandini, "Learning precise $3 \mathrm{~d}$ reaching in a humanoid robot," in International Conference of Development and Learning. London, UK: IEEE, 2007.

[19] S. Vijayakumar and S. Schaal, "Locally weighted projection regression : An o(n) algorithm for incremental real time learning in high dimensional space," in International Conference on Machine Learning (ICML), 2000.

[20] J. Chestnutt, J. Kuffner, K. Nishiwaki, and S. Kagami, "Planning biped navigation strategies in complex environments," in IEEE Int. Conf. Hum. Rob., Munich, Germany, 2003.

[21] J.-I. Yamaguchi, A. Takanishi, and I. Kato, "Development of a biped walking robot compensating for three-axis moment by trunk motion," in International Conference on Intelligent Robots and Systems, vol. 1. IEEE, 1993, pp. 561-566.

[22] Y. Ogura, T. Kataoka, K. Shimomura, H.-o. Lim, and A. Takanishi, "A novel method of biped walking pattern generation with predetermined knee joint motion," in Intelligent Robots and Systems, 2004.(IROS 2004). Proceedings. 2004 IEEE/RSJ International Conference on, vol. 3. IEEE, 2004, pp. 2831-2836.

[23] F. Zacharias, C. Borst, and G. Hirzinger, "Online generation of reachable grasps for dexterous manipulation using a representation of the reachable workspace," in International Conference on Advanced Robotics, 2009. 